AperTO - Archivio Istituzionale Open Access dell'Università di Torino

\title{
Rogue waves: a unique approach to multidisciplinary physics
}

\section{This is the author's manuscript}

Original Citation:

\section{Availability:}

This version is available http://hdl.handle.net/2318/1618714

since 2016-11-29T18:47:40Z

Published version:

DOI:10.1080/00107514.2016.1243351

Terms of use:

Open Access

Anyone can freely access the full text of works made available as "Open Access". Works made available under a Creative Commons license can be used according to the terms and conditions of said license. Use of all other works requires consent of the right holder (author or publisher) if not exempted from copyright protection by the applicable law. 



\title{
Rogue waves: a unique approach to multidisciplinary physics
}

\author{
S. Residori ${ }^{\mathrm{a}}$, M. Onorato ${ }^{\mathrm{b}, \mathrm{c}}$, U. Bortolozzo ${ }^{\mathrm{a}}$ and F. T. Arecchi ${ }^{\mathrm{d}, \mathrm{e}}$ \\ ${ }^{a}$ INLN, CNRS, Université de Nice-Sophia Antipolis, Valbonne, France; ${ }^{b}$ Dipartimento di Fisica, Università degli Studi di Torino, Torino, Italy;

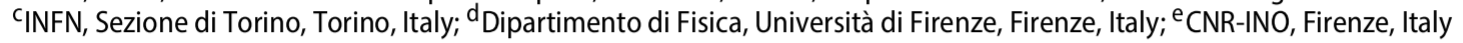

\begin{abstract}
Rogue waves are giant waves appearing erratically and unexpectedly on the ocean surfaces. Their existence, considered as mythical in the ancient times, has recently been recognised by the scientific community and, since then, rogue waves have become the object of numerous theoretical and experimental studies. Their relevance is not restricted to oceanography, but it extends in a wide spectrum of physical contexts. General models and mathematical tools have been developed on a interdisciplinary ground and many experiments have been specifically conceived for the observation of rogue waves in a variety of different physical systems. Rogue wave phenomena are, nowadays, studied, for instance, in hydrodynamics, optics, plasmas, complex media, Bose-Einstein condensation and acoustics. We can, therefore, consider rogue waves as a paradigmatic description, able to account for the manifestation of extreme events in multidisciplinary physics. In this review, we present the main physical concepts and mathematical tools for the description of rogue waves. We will refer mostly to examples from water waves and optics, the two domains having in common the non-linear Schrödinger equation from which prototype rogue wave solutions can be derived. We will highlight the most common features of the rogue wave phenomena, as the large deviations from the Gaussian statistics of the amplitude, the existence of many uncorrelated 'grains' of activity and their clustering in inhomogeneous spatial domains via large-scale symmetry breaking.
\end{abstract}

ARTICLE HISTORY

Received 9 December 2015 Accepted 26 September 2016

\section{KEYWORDS}

Rogue waves; non-linear Schröedinger equation; breathers and solitons; non-Gaussian statistics; water waves; optical fibers; non-linear optical cavities; multimode systems

\section{Introduction}

'Rogue waves' is the name that has been given to the erratic giant waves spontaneously occurring on the ocean surfaces. Reported in several stories about ocean storms, rogue waves were first considered as part of the maritime folklore, with narrations of almost vertical walls of water, as tall as $20-30 \mathrm{~m}$, suddenly appearing in the oceans and preceded by a trough so deep that it was indicated as a 'hole in the sea'. These extremely dangerous waves represent a tremendous experience for a ship encountering one of them and rogue waves, indeed, constitute a threat even for large boats or ocean liners. Nevertheless, despite the numerous witnesses of such extreme waves, the scientific community remained relatively silent, even sceptic, until the first 'measurement' of a rogue wave was reported. This was the famous Draupner wave, a single giant wave occurring at the Draupner platform in the North Sea off the coast of Norway on 1 January 1995 [1]. The elevation of the ocean surface was measured thanks to a downwards-pointing laser sensor. From the time series recorded by this optical sensor, it was possible to determine that the rogue wave reached a maximum height of $25.6 \mathrm{~m}$ over a significant wave height of almost $12 \mathrm{~m}$ (see Figure 1). The validity of the optical reading allowed to unambiguously confirm the existence of rogue waves, that were finally recognised as fundamental physical phenomena deserving all the attention from the scientific community. Since then several rogue wave events were measured in the oceans, among the most famous ones we can cite the Andrea wave [2], measured in the North Sea in 2007, or the one measured in 2002 three kilometres off the Yura fishing harbour facing the Sea of Japan [3]. Investigation of rogue waves in oceanography has, then, been constantly growing, with an increasing number of studies carried on both from the theoretical and the experimental side. At the same time, the frame of interest of rogue waves has exited the pure context of oceanography, extending in a wide spectrum of physical contexts. It is now accepted that rogue waves constitute genuine wave phenomena, their origin being of universal nature and their manifestation potentially occurring in any wave systems, whatever the nature of the waves considered.

Important contributions to the recent burst of investigations on rogue waves have come from the domain of optics. Triggered by the seminal work of Jalali group [4], where the concept was first introduced, optical rogue waves have become a subject of investigation on its own. 


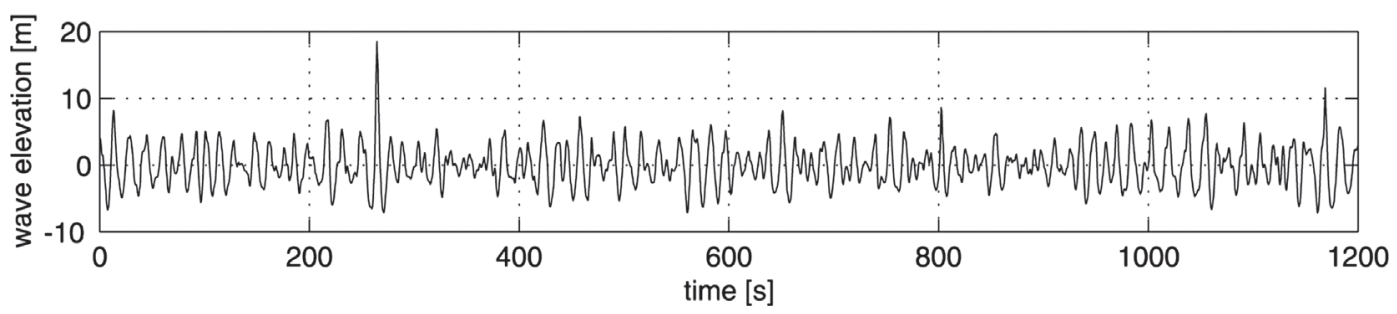

Figure 1. The time series of the Draupner rogue wave. The maximum wave height is approximately $25.6 \mathrm{~m}$ with a peak elevation of $18.5 \mathrm{~m}$.

First optical rogue waves have been identified with the high amplitude peaks occurring in the supercontinuum generated by a non-linear photonic crystal fibre [4], then, rogue waves have been reported in many different types of optical systems, see e.g. [5,6]. Rogue waves have also been reported in a large number of other physical domains, as in water waves, linear and non-linear optics, plasmas, superfluids, Bose-Einstein condensates, acoustics (see, e.g. [5] and references therein).

The aim of this review is to give an introduction to the basic physical concepts and mathematical tools required to describe rogue wave phenomena in a multidisciplinary context. Among the various experiments we will refer mostly to examples from water waves and optics, the two domains have in common one of the fundamental equations in non-linear physics, the nonlinear Schrödinger equation, NLSE, from which prototype rogue wave solutions can be derived. Numerical simulations also play an important role in shedding light on basically involved mechanisms, such as the modulational instability, emergence of large breathers and large filaments in wave turbulence. Here, we will present some of the most prominent features of the rogue wave phenomena, as the large deviations from the Gaussian statistics of the amplitude, the existence of many uncorrelated 'grains' of activity and their clustering in inhomogeneous spatial domains via large-scale symmetry breaking. As for an universal definition or a general classification in terms of different statistics and nature of the involved waves, this still constitutes the subject of ongoing researches and continues to stimulate a growing number of investigations.

Section 2 provides a brief introduction to NLSE and its applications both in the context of water waves and nonlinear optics. The localised breather solutions of the NLSE are also described in this section, as well as the superregular and higher order solutions which are considered as the rogue wave prototypes. In Section 3 are given several examples of laboratory experiments in which the NLSE solutions have been reproduced. Section 4 presents rogue waves as rational solutions of integrable partial differential equations, such as the Manakov system, which describes two coupled sea states propagating in different directions, so-called the crossing sea-state, a phenomenon conjectured at the origin of rogue waves in the oceans. Section 5 is dedicated to an introduction to the main statistical properties of rogue waves, such as the exceedance probability and the large-tailed probability distribution function (PDF) of the wave amplitude. Rogue waves in spatially extended optical systems are presented in Section 6, where it highlighted the stretched exponential character of the PDF of the light intensity. In Section 7 , optical rogue waves occurring in pattern-forming systems, such as photorefractive or Kerr media with optical feedback, are presented and, finally, Section 8 are the conclusions.

\section{The non-linear origin of rogue waves: the NLSE}

Non-linearity, even though statistically small in the ocean, is considered as playing a fundamental role in the ocean wave dynamics and, thus, is expected to have a major part in the origin of the rogue wave phenomena. In particular, two types of non-linearities can be associated to the sea surface elevation, the first one resulting from the presence of the so-called bound, or phase locked, modes, the second one deriving from the quasi-resonant interaction between free modes $[7,8]$. In a similar way, non-linearity in optics is well-known to arise because of mode coupling, or multiple wave interaction, in nonlinear media, which gives rise to focusing or defocusing behaviours. Both in hydrodynamics and in optics, the paradigm model for non-linear wave interaction is the non-linear Schrödinger equation, NLSE,

$$
i\left(\frac{\partial A}{\partial t}+c_{g} \frac{\partial A}{\partial x}\right)=\beta \frac{\partial^{2} A}{\partial x^{2}}+\alpha|A|^{2} A,
$$

where $A$ is the complex wave amplitude, $c_{g}=\partial \omega / \partial k$ is the group velocity, $\omega$ the wave frequency and $k$ the wave number, and $\beta$ and $\alpha$ are two constant coefficients that weight, respectively, the dispersive and non-linear terms. This equation is able to catch the main features of nonlinear wave dynamics, as the modulational instability, the formation of solitons and the appearance of rogue 
waves. Moreover, the NLSE is an integrable equation via the Inverse Scattering Transform and such property can be used to identify rogue waves in random wave trains [9].

The modulational instability, also known as the Benjamin-Feir or side-band instability, describes the exponential growth of an initially sinusoidal long wave perturbation of a plane wave. The non-linear stage of the modulational instability is described by exact solutions of the NLSE, known as Akhmediev breathers [10] and considered as prototypes of rogue waves [11]. Besides the Akhmediev breather, the NLSE has a variety of solutions that are the results of a focusing process, among which the Kuznetsov-Ma breather [19,20], the Peregrine soliton [12], the $N$-breather solution [13] and the superregular breathers [14].

In hydrodynamics, the coefficients $\alpha, \beta$ and $c_{g}$ are, in general, complicated functions of the carrier wave number $k_{0}$, the water depth $h$ and the gravity acceleration $g$. However, in the limit of infinite water depth, the coefficients reduce notably to $c_{g}=\omega_{0} /\left(2 k_{0}\right), \alpha=\omega_{0} k_{0}^{2} / 2$ and $\beta=\omega_{0} /\left(8 k_{0}^{2}\right)$ with $\omega_{0}=\sqrt{g k_{0}}$. In this limit, it is possible to introduce a dispersive, $L_{D}$, and a nonlinear, $L_{N L}$, length as follows, $L_{D}=\left(\omega_{0}^{2} / \Delta \Omega^{2}\right) / k_{0}$ and $L_{N L}=1 /\left(\left|A_{0}\right|^{2} k_{0}^{3}\right)$, where $\Delta \Omega$ is a measure of the spectral bandwidth and $\left|A_{0}\right|$ is a typical wave amplitude of the considered process. The ratio between the dispersive to the non-linear length is known as the Benjamin-Feir Index (BFI) [15,16]

$$
\mathrm{BFI}=\sqrt{\frac{L_{D}}{L_{N L}}}=\frac{\left|A_{0}\right| k_{0}}{\Delta \Omega / \omega_{0}},
$$

a non-dimensional parameter related to the probability of formation of rogue waves in incoherent wave trains. In the context of water waves, in particular, in oceanography, the BFI is used as an indicator for rogue wave forecasting, as the probability of finding extreme waves increases for initial conditions characterised by large BFI.

In the context of optics the NLSE is used, for instance, to describe optical pulse propagation in non-linear fibres. In this context, it is usually written in the form (see, e.g. [17])

$$
i \frac{\partial \psi}{\partial x}-\frac{\beta_{2}}{2} \frac{\partial^{2} \psi}{\partial t^{2}}+\frac{\omega_{0}}{c} n_{2}|\psi|^{2} \psi=0,
$$

where space takes the role of time, $\beta_{2}=\left(\partial^{2} k / \partial \omega^{2}\right)_{\omega_{0}}$ is the group velocity dispersion coefficient, $\omega_{0}$ the central frequency of the pulse and $n_{2}$ the non-linear coefficient expressing the Kerr-like optical response of the medium. The pulse evolution can be characterised by two characteristic lengths, the dispersive length $L_{D}^{-1}=\left|\beta_{2}\right| / 2 \delta_{0}^{2}$, where $\delta_{0}$ is the time width of the input pulse, and the non-linear length $L_{N L}=\omega_{0} n_{2} /\left(c P_{0}\right)$, where $P_{0}$ is the peak pulse amplitude. A non-dimensional number that weights the linear to the non-linear contribution, $N \equiv$ $L_{D} / L_{N L}$ can be introduced. The interplay between group velocity dispersion and self phase modulations is ruled by $N$, with non-linear effects becoming dominant at large $N$ and soliton balance between dispersion and non-linear focusing occurring for $L_{D} \sim L_{N L}$. For this reason $N$ is also called the soliton number.

A detailed discussion on the analogy between NLSE in non-linear optics and in water waves can be found in [18]. In particular, in analogy with water waves, the BFI can be derived for optical fibres characterised by a Kerr non-linearity, which takes the form

$$
\mathrm{BFI}_{K}=\frac{\sqrt{\frac{2 n_{2}\left\langle|A|^{2}\right\rangle}{\omega_{0} c\left|\beta_{2}\right| S_{e f f}}}}{\Delta \Omega / \omega}
$$

where $S_{\text {eff }}$ is the effective area of the fibre core, $\left\langle|A|^{2}\right\rangle$ is the optical power and $\Delta \Omega / \omega_{0}$ is the relative frequency spectral bandwidth. The $\mathrm{BFI}_{K}$ can also be constructed as the ratio of the dispersive to non-linear length, $\mathrm{BFI}_{K}=$ $\sqrt{L_{D} / L_{N L}}$ in analogy with the water waves BFI, and provides an indicator of the probability of occurrence of optical rogue waves.

The NLSE exhibits localised solutions, solitons or breathers type, which are considered as the prototypes of rogue waves. Examples of the classical NLSE localised solutions are shown in Figure 2. By taking the conventional form of NLSE for water waves, Equation (1), in a frame of reference moving with the group velocity, the Akhmediev breather is a solution periodic in space $x$ and localised in time $t$, which describes the modulational instability in its non-linear regime [10] and has the following analytical form:

$$
\begin{aligned}
A(x, t)= & A_{0} \exp \left[-i \beta A_{0}^{2} t\right] \\
& \times\left(\frac{\left.\sqrt{2} \tilde{v}^{2} \cosh [\Omega t]-i \sqrt{2} \tilde{\sigma} \sinh [\Omega t)\right]}{\sqrt{2} \cosh [\Omega t]-\sqrt{2-\tilde{v}^{2}} \cos [K x]}-1\right),
\end{aligned}
$$

where $\tilde{v}=K / A_{0} \sqrt{\alpha / \beta}, \tilde{\sigma}=\tilde{v} \sqrt{2-\tilde{v}^{2}}, \Omega=\beta A_{0}^{2} \tilde{\sigma}$, and $K$ is the wave number of the perturbation. For large negative times the solution becomes $A_{0} \exp (i \phi)(1+\delta \cos$ $(K x)$ ) with $\delta$ small, which corresponds to a perturbed Stokes wave. $\Omega$ is the exponential growth rate of the perturbation. The perturbation grows if $2-\tilde{v}^{2}>0$, which for deep water waves corresponds to the condition $K<2 \sqrt{2} A_{0} k_{0}^{2}$. The absolute maximum of the Akhmediev breather, $A_{\max }$, can be found and it can be shown that 

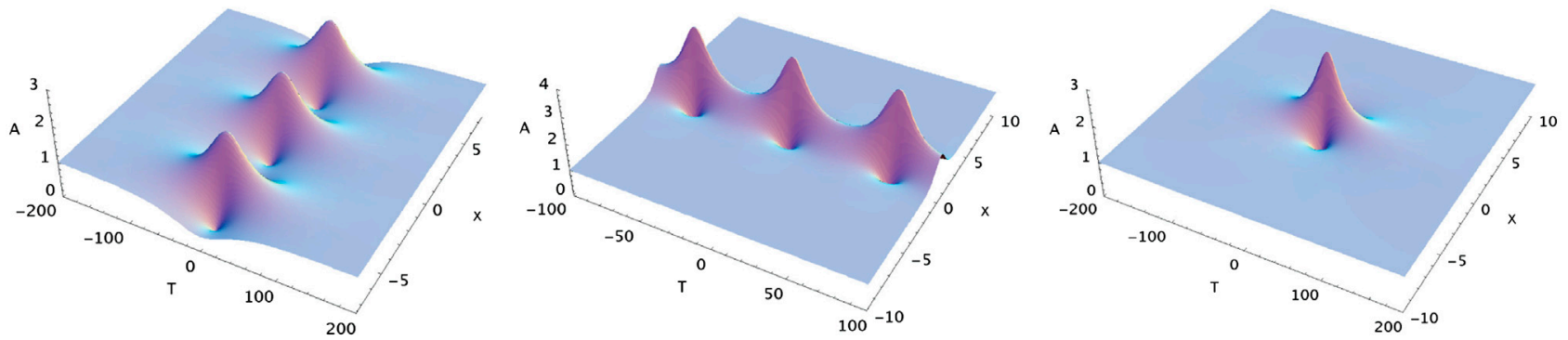

Figure 2. The localised solutions of NLSE : (a) Akhmediev breather, (b) KM solution, (c) Peregrine soliton. In all the plots the time is non-dimensionalised by the wave period, the space by the wavelength and the amplitude by $A_{0}$; only the wave envelope is shown.

$$
\frac{A_{\max }}{A_{0}}=1+2 \sqrt{1-\frac{\tilde{\nu}^{2}}{2}}=1+2 \sqrt{1-\left(\frac{1}{2 \sqrt{2} \epsilon k_{0} / K}\right)^{2}} .
$$

The maximum amplitude is reached when $\epsilon k_{0} / K \rightarrow \infty$, and for such case $A_{\max } / A_{0}=3$, which corresponds to the Peregrine solution.

The Kusnetzov-Ma (KM) solution is periodic in time $t$ and localised in space $x[19,20]$. While for the Akhmediev breather the large time limit is a plane wave plus a small perturbation, the modulation for the KM breather is never small; therefore it does not corresponds to the classical Benjamin-Feir instability. The solution has the analytical form

$$
\begin{aligned}
A(x, t)= & A_{0} \exp \left[-i \beta A_{0}^{2} t\right] \\
& \times\left(\frac{\left.-\sqrt{2} \tilde{\mu}^{2} \cos [\Omega t]+i \sqrt{2} \tilde{\rho} \sin [\Omega t)\right]}{\sqrt{2} \cos [\Omega t]-\sqrt{2+\tilde{\mu}^{2}} \cosh [K x]}-1\right),
\end{aligned}
$$

with $\tilde{\mu}=K / A_{0} \sqrt{\alpha / \beta}, \tilde{\rho}=\tilde{\mu} \sqrt{2+\tilde{\mu}^{2}}, \Omega=\beta A_{0}^{2} \tilde{\rho}$. In a similar way as for the Akhmediev breather one can find the maximum amplitude and obtain

$$
\frac{A_{\max }}{A_{0}}=1+\sqrt{2} \sqrt{2+\tilde{\mu}^{2}}=1+\sqrt{2} \sqrt{2+\left(\frac{1}{2 \epsilon k_{0} / K}\right)^{2}} .
$$

The Peregrine solution, also known as rational solution, has been originally proposed in [12]. It has the peculiarity of being localised both in time $t$ and in space $x$ and, therefore, it can be identifies as a wave that 'appears from nowhere and disappears without trace' [21]. Besides, its maximum amplitude reaches three times the amplitude of the unperturbed waves. For these reasons it has been considered as a special prototype of rogue waves [22]. The analytical form of the Peregrine solution is

$$
\begin{aligned}
A(x, t)= & A_{0} \exp \left[-i \beta A_{0}^{2} t\right] \\
& \times\left(\frac{4 \alpha\left(1-i 2 \beta A_{0}^{2} t\right)}{\alpha+\alpha\left(2 \beta A_{0}^{2} t\right)^{2}+2 \beta A_{0}^{2} x^{2}},-1\right),
\end{aligned}
$$

where $\alpha$ and $\beta$ are, respectively, the coefficient of the dispersive and non-linear term in (1), i.e. in infinite water depth $\alpha=\omega_{0} /\left(8 k_{0}^{2}\right)$ and $\beta=k_{0} \omega_{0}^{2} / 2$.

It is worth noting that the breather solutions are related one to the other. Their properties are such that simple transformations link a solution to the other (see, e.g. [23]). For instance, the Akhmediev breather becomes the KM solution if $K$ is replaced by $i K$. Both the Akhmediev breather and the KM breather become the Peregrine soliton in the limit of $K \rightarrow 0$. The Akhmediev breather becomes the plane wave solution if $\tilde{v}=\sqrt{2}$ and the KM breather becomes a soliton in the limit of $K \rightarrow \infty$.

\subsection{Superregular and higher order breather solutions}

A new family of exact solutions of NLSE have been derived in [14], which are named 'superregular breathers' and can be used as rogue wave prototypes. They describe a generalised modulation instability that develops from localised perturbations of a plane wave. As for the Akhmediev breathers, the standard modulational instability is obtained from an infinitesimal perturbation of the coherent background, however, the perturbation, here, is localised and not periodic. The superregular breathers can be obtained as a non-linear superposition of $n$ pairs, with $n$ integer, of quasi-Akhmediev breathers propagating in opposite directions. Such mathematical objects are able to describe different configurations of the non-linear stage of the modulational instability, such as the amplification, annihilation or ghost interaction of localised perturbations.

The mathematical expressions for the superregular breathers are complicated functions of a number of free parameters [24] and are not reported here. To give a qualitative example, we show in Figure 3 a plot of such a superregular solution taken from [25]. It can be noticed the higher steepness and higher amplitude of the central peak of this solution when compared to the breather solutions shown in Figure 2. 


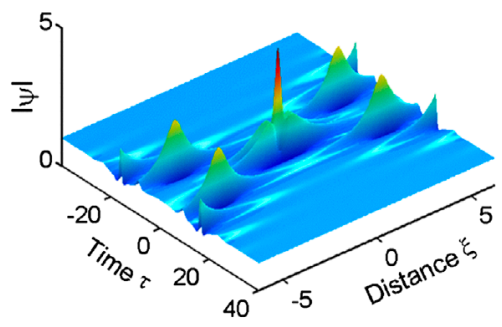

Figure 3. One-pair breather solution of the focusing NLSE: the superregular breather. From [25].

In general, it is possible to build non-linear superpositions of breathers to generate NLSE solutions. The solutions associated with this mechanism are known as higher order breathers and display higher amplitude peaks with respect to the building-block breathers, the amplification factor increasing with the order of the solution. These solutions attract a lot of attention as rogue wave prototypes [13,26-28]. In [21], a method for finding the hierarchy of rational solutions of the self-focusing NLSE is proposed. The process starts by seeding the evolution equation with simple solutions, such as plane waves, then, the higher order solutions are constructed using the recursive Darboux method (see, e.g. [29] for a detailed presentation of the method). The fundamental component, or elementary building block of a rogue wave, is a first-order breather, and starting from this all higher order solutions are constructed.

A non-linear superposition of order $n$ requires $n$ firstorder components. It can be shown that, if a label $j$ is given to each component this can be translated by an amount $x_{j}$ or $t_{j}$ along the $x$ or $t$ axes, respectively. Different symmetrical arrangements of localised peaks are obtained depending on the amount of temporal and spatial translation. In Figure 4 it is shown the hierarchy of the fundamental NLSE rogue-wave solutions up to order 6. Circular clusters are obtained on the diagonal of the space-time translation diagram. In the rogue wave limit, the solutions appear as patterns of peaks, each peak corresponding to a Peregrine soliton or a combination of them. The peak amplitude increases with the order of the solution. If the amplitude of the seeding plane-wave is fixed to 1 , the maximum amplitude reached, for instance, by the fourth-order solution is 9 . It appears from a mathematical point of view that there is no limitation in the amplification factor of such solutions.

\section{Laboratory experiments and NLSE solutions as rogue wave prototypes}

Several localised solutions of the NLSE have been faithfully reproduced in laboratory experiments in order to demonstrate the non-linear origin of the rogue wave phenomena. The elementary building-blocks of the rogue waves as well as several higher order rational solutions have been reproduced in a controlled way. Experiments were mainly in fluid dynamics and in optics, the analogies between the two approaches stemming from the qualitative similarity of water waves propagating in a channel and light pulses travelling in an optical fibre. NLSE solutions have been recreated in laboratory by imposing appropriate initial conditions for water waves in a tank [30] and by using appropriately addressed input beams in optical fibres [35].

\subsection{Water waves: breathers and higher order solutions}

The first demonstration of a localised solution of NLSE was the Peregrine soliton obtained in wave tank experiments [30]. The set-up consists of a $15 \times 1.6 \times 1.5 \mathrm{~m}$ tank with $1 \mathrm{~m}$ water depth. The appropriate initial conditions are realised using a single-flap paddle activated by a hydraulic cylinder to generate the waves at the one end of the channel. A wave-absorbing beach is located at the opposite end of the tank in order to avoid wave reflections. The wave height is measured at a given point by a capacitance wave gauge with a sensitivity of $1.06 \mathrm{~V} / \mathrm{cm}$ and a sampling frequency of $500 \mathrm{~Hz}$.

The Peregrine soliton is located on a background wave which provides energy for its growth. The choice for the parameters of this wave is dictated by the size of the tank and the position where the rogue wave develops its maximum amplitude depends on the initial conditions at the wave maker. By setting appropriate parameters, the maximum breather amplitude was excited at about $9 \mathrm{~m}$ along the tank. The water surface-height data have been collected at 10 positions, with equal separations of $1 \mathrm{~m}$, along the direction of wave propagation. An example of the experimentally recorded Peregrine soliton is displayed in Figure 5. The carrier wave has an amplitude of about one centimetre and the maximum surface height of the breather reaches a value of three centimetres. For comparison, the measured time series (solid line) is plotted together with the curve predicted by the Peregrine soliton solution (dashed line), showing a good agreement between theory and experiment. It is worth noting that such a Peregrine soliton has been used experimentally to study the impact of a rogue wave on a ship [31].

The Kuznetsov-Ma solution has also been reproduced in wave tank experiments [33], as well as higher order solutions $[32,34]$. The hierarchy of rational breather solutions up to order five have been successfully generated for appropriate initial conditions obtained with a single-flap paddle to generate the waves at one end of the 


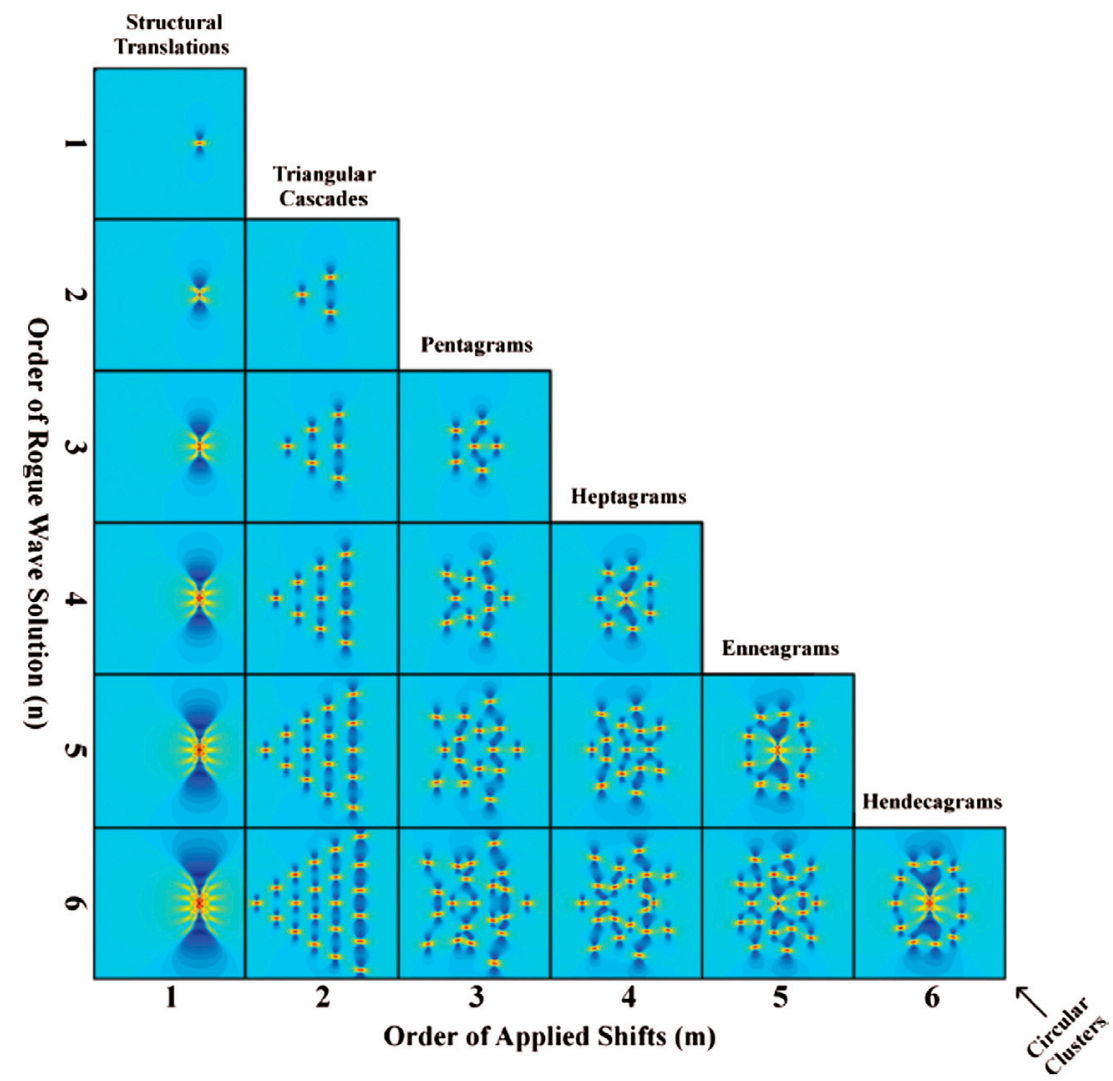

Figure 4. Fundamental rogue wave solutions up to order 6 . Structures belonging to column $m$ (for $m>1$ ) have effective radial symmetry of order $2 m-1$. Each circular cluster along the diagonal $(n=m)$ displays a ring of $2 m-1$ Peregrine solitons around a central rogue wave of order $m-2$ (for $m>2$ ). Reproduced with permission from [29].

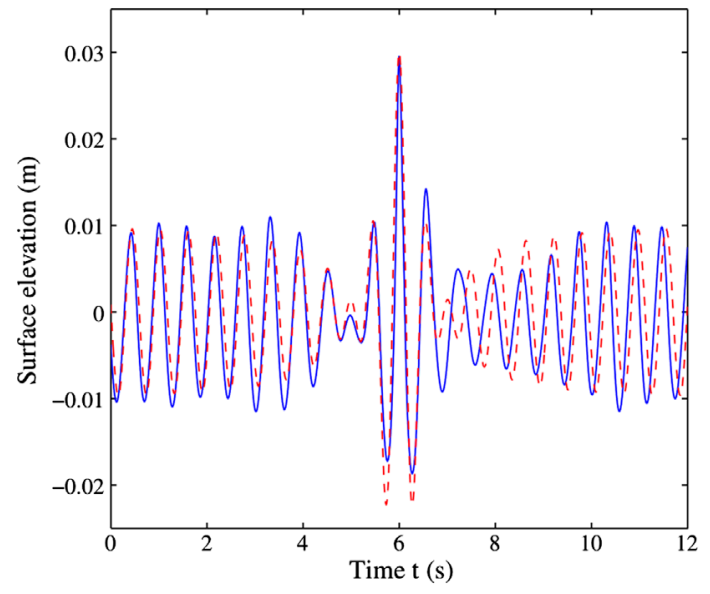

Figure 5. Peregrine soliton obtained in water tank experiments: measured surface height (solid line) and theoretically calculated Peregrine solution (dashed line). Reproduced with permission from [30].

channel. A second-order rational solution obtained in the water tank experiment is shown in Figure 6. A maximal amplification of a factor 5 above the background wave has been obtained, in good agreement with the theoretical prediction. More recently, a superregular breather has also been successfully reproduced [25]. An example of experimental observation together with the comparison with the theoretical prediction is shown in Figure 7.

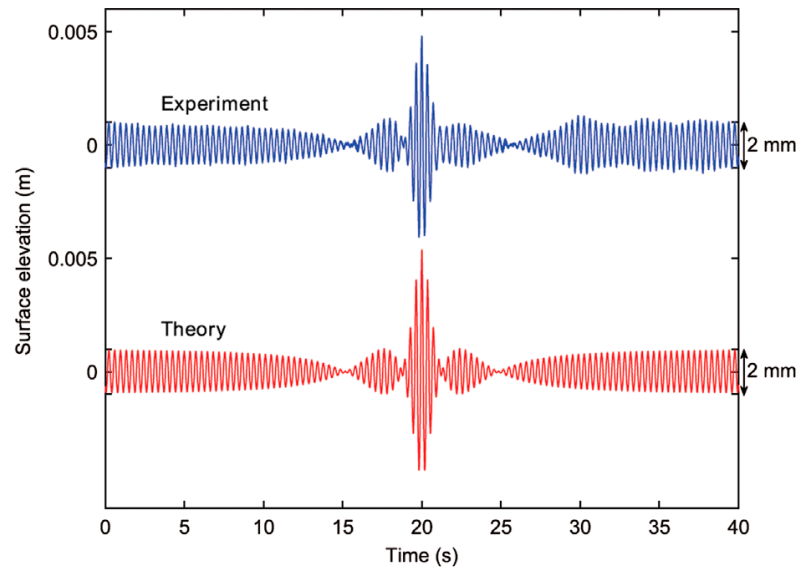

Figure 6. Second-order rational solution of the NLSE obtained in water tank experiments (upper curve) and comparison with the theoretical solution (lower curve); the maximal amplification above the background wave is 5. From [32].

\subsection{Optical fibres: breathers and higher order solutions}

Peregrine solitons have been experimentally realised in non-linear optical fibres generating femtosecond pulses [35]. The NLSE equation describing the pulse propagation in the fibre can be written as 
(a) Experiment

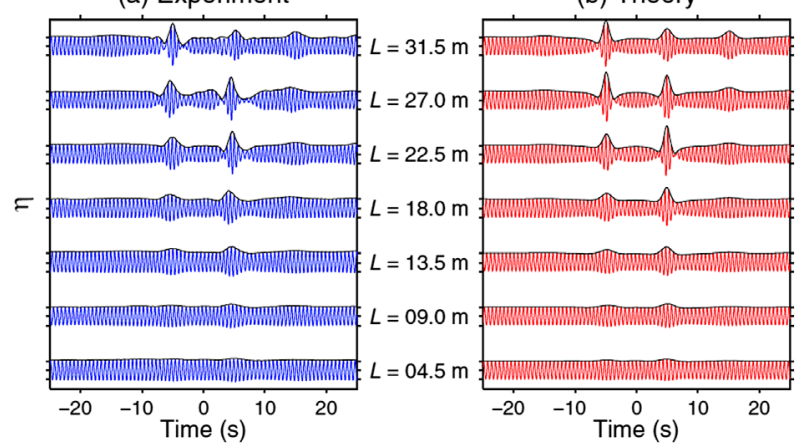

Figure 7. Creation of a pair of breathers: (a) water wave experiments and (b) analytical solutions. The envelopes, in black, are calculated using the Hilbert transform. From [25].

$$
i \frac{\partial A}{\partial z}-\frac{\beta_{2}}{2} \frac{\partial^{2} A}{\partial T^{2}}+\gamma|A|^{2} A=0
$$

where $A(z, T)$ is the field envelope, $\beta_{2}<0$ is the group velocity dispersion and $\gamma$ is the non-linear coefficient. The input field can be expressed as $A\left(z=z_{0}, T\right)=$ $\sqrt{P_{0}}\left[1+\alpha_{\bmod } e^{\left(i \omega_{\bmod } T\right)}\right]$, with $P_{0}$ the input power, $\alpha_{\bmod }$ and $\omega_{\text {mod }}$ the modulation strength and frequency, respectively. By defining the non-linear length $L_{N L}=\left(\gamma P_{0}\right)^{-1}$ and the timescale $T_{0}=\left(\left|\beta_{2}\right| L_{N L}\right)^{1 / 2}$, the dimensional distance $z$ and time $T$ are related to the normalised parameters of the non-dimensional NLSE by the expression $z=x l_{N L}$ and $T=t T_{0}$. Given these definitions, it can be seen that a modulational instability occurs for $\omega_{\text {mod }} \rightarrow 0$, which is accessible in practice by beating two narrow-linewidth lasers to create an initial lowfrequency-modulated wave. The input field was obtained from a pump laser at $\lambda_{p}=1.55 \mu \mathrm{m}$ mixed with a closely spaced tunable signal at $\lambda_{s}$, with $\alpha_{\bmod }=0.225$. The Peregrine soliton was found in the divergent regime at low-frequency modulation and at maximum pulse compression.

The dynamics of Akhmediev breathers has also been studied in an optical fibre with a longitudinally tailored dispersion, which has allowed to nearly freeze the evolution of the breathers near their point of maximal compression [36]. The results show a good agreement with numerical simulations of the modified NLSE. Then, using a similar set-up, the KM soliton was obtained. Highspeed telecommunications-grade components were used to strongly modulate the output of a continuous laser diode at $\lambda_{p}=1.55 \mu \mathrm{m}$. The modulation strength and period were chosen such that the characteristics of each cycle match a particular KM soliton. The experimental results are shown in Figure 8, where the evolution of the peak power is displayed (Figure 8 (a) as a function of the normalised propagation distance along the fibre $z / z_{p}$, where $z_{p}$ corresponds to one period of the KM cycle, and as a function to time (Figure $8(\mathrm{~b})$. The frequency spectrum of the KM soliton is shown for the maximum temporal compression at $z_{p} / 2$ (Figure 8(c). Again, a good qualitative agreement with the theoretical solution can be appreciated.

More recently, the development of a small localised perturbation into a pair of breathers for two propagation distances have been observed in a similar set-up [25]. Comparison between theory and experiments is shown in Figure 9. Remarkably, the similar qualitative behaviour with the superregular breathers observed in water waves (Figure 7) can be appreciated.

Finally, watch-hand-like super rogue wave patterns have recently been found as high-order solutions of the equations that govern the resonant interaction of three optical pulses in quadratic non-linear media [38]. These rogue wave solutions exhibit a peak amplitude more than five times the background height and are attributed to the non-linear superposition of six Peregrine solitons.

\section{Rogue waves as rational solutions of PDEs}

The Peregrine solution, which is the first prototype of a rogue wave, corresponds to a rational solution of the standard NLSE. In the recent years, an important research activity has been developed in order to find rational solutions of integrable partial differential equations. In this framework, rational solutions are called 'rogue waves'. As a first example, the following coupled nonlinear Schrödinger equations, called Manakov system, has been analysed

$$
\begin{aligned}
& i u_{t}+u_{x x}+2\left(|u|^{2} \pm|v|^{2}\right) u=0 \\
& i v_{t}+v_{x x}+2\left(|v|^{2} \pm|u|^{2}\right) v=0
\end{aligned}
$$

where the index indicates derivatives. The interest for this system has been triggered by the fact that in ocean waves there has been some conjectures on the formation of rogue waves in the presence of crossing seas, i.e. two sea states propagating in different directions [39-44], such sea states being described by a non-integrable version of the system, Equation (11). In the focusing regime (' + ' sign) a semi-rational, multiparametric vector solution is derived, which includes the vector Peregrine solutions, bright- and dark-rogue solutions and novel vector rogue waves, which features both exponential and rational dependence on coordinates [45]. Solutions are found by using the Darboux dressing technique. In [46] are reported rogue wave solutions of the defocusing vector NLSE ('-' sign in Equation (11)). This family of solutions includes bright-dark and dark-dark rogue waves. The link between modulational instability and rogue waves is displayed by showing that only the so-called baseband instability can sustain rogue-wave formation [47]. 

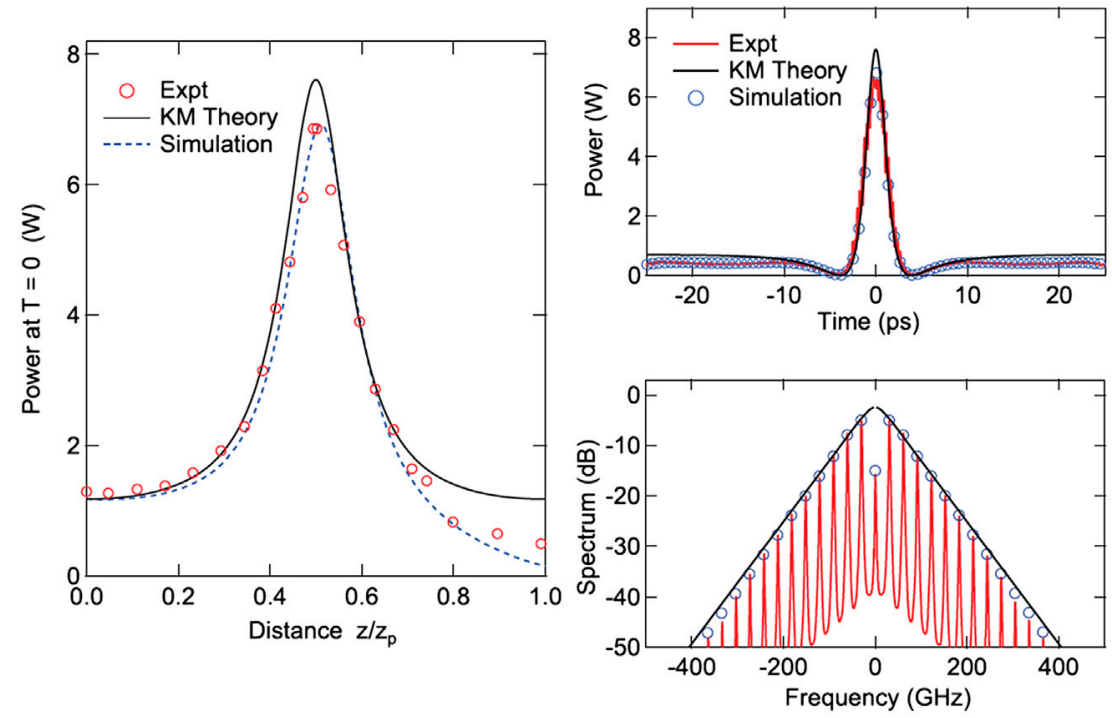

Figure 8. Experimental observation of KM soliton in optical fibres. (a) Evolution of the peak power as a function of the normalised distance $z / z_{p}$; (b) temporal evolution; and (c) frequency spectrum of the KM soliton for the maximum temporal compression at $z_{p} / 2$. The experimental data (red) are compared with the theoretical evolution (black) and simulation (blue). Reproduced with permission from [37].

(a) Experiment
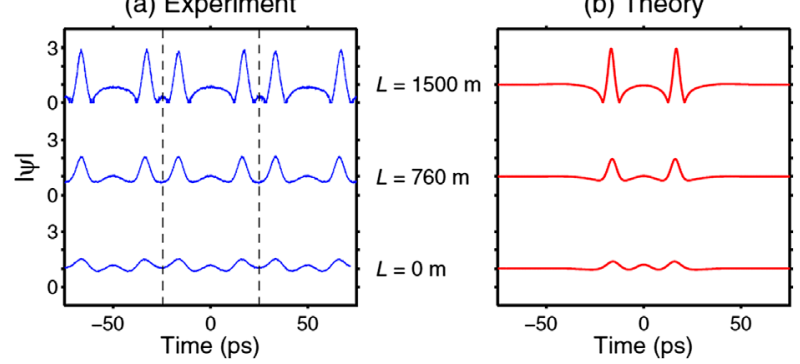

Figure 9. Superregular breathers in optical fibers: (a) experiments and (b) theory. Only the wave envelopes are shown. From [25].

Other examples of rational solutions of an integrable system can be found in [48], where a novel family of analytic solutions of three-wave resonant interaction equations at the purpose of modelling rogue waves is introduced. The system of equations has the following form

$$
\begin{aligned}
& u_{t}+c_{1} u_{x}=v^{*} w^{*} \\
& v_{t}+c_{2} v_{x}=-u^{*} w^{*} \\
& w_{t}+c_{3} w_{x}=u^{*} v^{*},
\end{aligned}
$$

where $c_{1}, c_{2}, c_{3}$ are the group velocities of the three complex wave amplitudes $u, v, w$. Applications of the above equations can be found in fluid dynamics (capillary-gravity waves, internal gravity waves, surface and internal waves), in optics (parametric amplification, frequency conversion, stimulated Raman and Brillouin scattering), in plasmas (plasma instability, laser-plasma interactions, radio frequency heating), in acoustics and solid-state physics.
Then, the long-wave-short-wave equation

$$
\begin{aligned}
& i u_{t}+\frac{1}{2} u_{x x}+u v=0 \\
& v_{t}-\left(|u|^{2}\right)_{x}=0,
\end{aligned}
$$

has been introduced and discussed in terms of exact rogue wave solutions in [49]. Here, $u$ is a complex field that describes the envelope of the short waves, while $v$ is a real long-wave field. The system has applications in plasma physics, non-linear optics, hydrodynamics, particularly in the capillary-gravity waves interaction. By following the standard Darboux dressing procedure, the fundamental rogue wave solution has been found. A similar solution, using the Hirota bilinear form of Equation (13), has also been found in [50].

\section{Basics of rogue wave statistics: exceedance probability and large tailed PDF}

The most salient feature of rogue waves statistics is that the PDF of the wave amplitude deviates from the Gaussian probability expected from the central limit theorem when a large number of uncorrelated events are recorded. Indeed, ocean waves are usually treated as homogeneous and stationary stochastic processes characterised by Fourier phases uniformly distributed in the $[0,2 \pi]$ interval $[51,52]$. In the linear approximation, the wave components are assumed to be independent from each other, then, according to the central limit theorem, the surface elevation should be characterised by a Gaussian PDF. However, rogue waves and non-linear effects lead to deviations from the Gaussian behaviour. 
Under the hypothesis of small amplitude, the surface elevation is described in oceanography by a Gaussian distribution. Correspondingly, the PDF of the wave envelope $|A|$ is given by

$$
p(|A|)=\frac{|A|}{\sigma^{2}} \exp \left[-\frac{|A|^{2}}{2 \sigma^{2}}\right],
$$

where $\sigma$ is the standard deviation of the surface elevation. The above PDF, Equation (14), is called the Rayleigh distribution. In practice, oceanographers prefer to compute the statistics of wave heights $H$, where $H$ is the difference between the maximum of the surface elevation in between two zero-crossings and the minimum of the surface elevation in the adjacent zero crossing interval (see, e.g. [5]). For a linear and narrow band process, $H \simeq 2|A|$, then, the statistics of the wave height is closely related to the envelope statistics.

Another currently used concept is the significant wave height, $H_{s}$, which expresses the wave height estimated by a 'trained observer' [53]. Nowadays, it is a common rule to identify the significant wave height with $4 \sigma$, that is, $H_{s}=4 \sigma$. Finally, often one is interested in addressing the problem on what is the probability of encountering a wave whose height is larger than some specific height $H_{0}$, i.e the exceedance probability defined as

$$
\begin{aligned}
S\left(H>H_{0}\right) & =\int_{H_{0}}^{\infty} \frac{H}{4 \sigma^{2}} \exp \left[-\frac{H^{2}}{8 \sigma^{2}}\right] \mathrm{d} H \\
& =\exp \left[-\frac{H_{0}^{2}}{8 \sigma^{2}}\right]=\exp \left[-2 \frac{H_{0}^{2}}{H_{s}^{2}}\right] .
\end{aligned}
$$

This distribution function is particularly relevant in view of providing a statistical definition of rogue waves. For instance, sometimes in the literature, a rogue wave is defined for having a height such that $H>2 H_{s}$. However, this must be taken not as an universal definition but more as a qualitative indicator.

The comparison of the statistics for water waves and for optical waves is not straightforward because optical measurements do not provide direct access to the optical field. Indeed, the measured quantity is the light intensity, hence, the statistics must be defined for the intensity of the optical field. For independent linear waves, it is wellknown that they are Gaussian distributed for the field amplitude, which provides an exponential PDF for the intensity [54]. In the paper by Solli and co-workers [4] optical rogue waves were identified as high amplitude peaks in the time series of the light intensity at the output of a non-linear fibre. The rogue wave character of the observations was highlighted by the L shape of the intensity histograms, showing clearly large deviations from the Gaussian behaviour.
More recently, an optical fibre experiment has been developed in order to investigate integrable turbulence in the focusing regime of the one-dimensional NLSE [55], showing the appearance of rogue waves. In analogy with the broad spectrum excitation used in one-dimensional experiments for water waves, a random initial wave was launched in a single-mode optical fibre. The PDF of the optical power was measured using a specifically developed optical sampling set-up and found to evolve from the normal law to a strong heavy-tailed distribution, thus revealing the formation of rogue waves. Using an ultrafast acquisition system equivalent to microscope in the time domain, in [56] the direct observation of rogue waves in incoherent light on the time scale of pico seconds was achieved.

Also in the integrable turbulence regime, the dynamics of an incoherently pumped passive optical fibre ring cavity nearby the zero-dispersion wavelength of the fibre has been studied experimentally and numerically in [57]. The authors show that the cavity exhibits a quasisoliton turbulence dynamics, whose properties are controlled by the degree of coherence of the injected pump wave. As the coherence of the pump is reduced, a transition from the quasisoliton condensation regime towards the weakly non-linear turbulent regime characterised by short-lived rogue wave events is observed.

For equations of the NLS type an exact relation between the evolution of the fourth-order moment of the probability distribution and the evolution of the spectral broadening has been found in [58]. Such relation implies that an increase in the spectral bandwidth in the focusing/defocusing regime leads to an increase/decrease in the probability of formation of rogue waves. Indeed, in [59] it has been shown numerically that out of equilibrium spectra can lead to an increase in the kurtosis in deep water waves.

Note that in all the statistical approaches currently adopted, the systems under analysis are considered to be autonomous, that is, are considered to be well described by equations whose coefficients do not depend explicitly on time (or space). Non-autonomous problems are usually less treated in the rogue wave regime, even though this analysis could be interesting in revealing new phenomena, as the PDF could, in such cases, change in the course of time (or space).

\section{Rogue waves in spatially extended optical systems: the stretched exponential PDF}

Rogue waves in two-dimensional spatially extended optical systems have been studied in a non-linear optical cavity, a system characterised by non-linearity and presence of many transverse modes $[60,61]$. Here, rogue waves 
appear as high amplitude pulses that spontaneously build up on the transverse profile of the beam circulating in the cavity. The cavity is an unidirectional ring oscillator formed by three high-reflectivity dielectric mirrors and containing a liquid crystal light-valve, LCLV, which supplies the gain needed to overcome losses from the mirrors. The LCLV, formed by the assembly of a nematic liquid crystal layer with a photoconductive crystal, is pumped by an incoming laser beam, so-called pump beam, field amplitude $E_{p}$, optical wavelength $\lambda=532 \mathrm{~nm}$, enlarged and collimated (10 $\mathrm{mm}$ diameter), from which photons are injected in the cavity through two wavemixing processes occurring in the liquid crystal layer [60]. The total cavity length is $L=273.3 \mathrm{~cm}$ and a lens of $f=70 \mathrm{~cm}$ focal length is positioned at a distance $L_{1}=88.1 \mathrm{~cm}$ from the entrance plane of the LCLV in order to ensure the stability of the cavity modes. The coordinate system is taken such that $z$ is along the cavity axis and $x, y$ are on the transverse plane, where is the wavefront of the field $E_{c}$ circulating in the cavity. $E_{c}$ is spontaneously generated starting from the amplification of refractive index fluctuations in the liquid crystal layer.

While for low pump intensity the amplitude of the cavity field follows a Gaussian statistics, hence, an exponential PDF of the light intensity, for high pump, the system displays the emergence of spatiotemporal pulses with much higher amplitude with respect to the background. Such extreme events populate the tail of the PDF providing large deviations from Gaussianity $[5,61]$. The spatiotemporal pulses develop erratically in time and in space and live for a typical time that is dictated by the response time of the LCLV [60]. The features of the optical rogue wave statistics are characterised by measuring the PDF of the light intensity for increasing levels of the pump beam intensity $I_{p}$, corresponding to the passage from low to high non-linearity. In Figure 10(a) the PDF of the cavity field intensity, $I=\left|E_{c}\right|^{2}$, are displayed for $I_{p} / I_{t h}=1.8,4.0,4.2$ and 6.4 , where $I_{\text {th }}=1.2 \mathrm{~mW} / \mathrm{cm}^{2}$ is the threshold for the optical oscillations to start in the cavity. Increasingly, large deviations from the exponential behaviour are observed as $I_{p}$ is increased. Moreover, all the distributions are well fitted by the stretched exponential function

$$
P(I)=N e^{-\sqrt{c_{1}+c_{2} I}},
$$

where $1 / c_{1}$ is a parameter that measures the deviation from the exponential behaviour. Meanwhile that the PDF develops a large tail an increasing number of large amplitude peaks populates the optical wavefront over a specklelike lower amplitude background (see Figure 10(b)).

Here, the small spatial scale is given by non-linearity that yields transverse spatial grains of average size $\lambda L / a$, where $L$ is the free propagation length and $a$ is the size of the most limiting aperture in the cavity. Moreover, a large-scale inhomogeneity is introduced by the chosen geometrical configuration of the resonator. Indeed, because of the geometry of the cavity, which is formed by three mirrors in a quasi-spherical configuration, there is an inversion symmetry around the $y$ axis, hence, the field returning to the LCLV is inverted along $y$ after a round trip. This symmetry breaking provides a nonlocal coupling between distant domains. As a consequence, the cavity field $E_{c}$ develops into large-scale clusters of elementary grains that are dynamically coupled in a nonlocal way.

Numerical simulations have been performed using the full model equations of the cavity [62]

$$
\begin{aligned}
& \frac{\partial n_{0}}{\partial t}=-n_{0}+\alpha\left|E_{c}\right|^{2}, \\
& \frac{\partial n_{1}}{\partial t}=-n_{1}+\alpha E_{c} E_{p}^{*},
\end{aligned}
$$

where $n_{0}$ and $n_{1}$ are, respectively, the amplitude of the homogeneous refractive index and the amplitude of the photo-induced refractive index grating at the spatial frequency $k_{c}-k_{p}$, where $k_{c}$ and $k_{p}$ are the optical wave numbers of the pump $E_{p}$, and cavity field $E_{c}$, respectively. $\alpha$ is the non-linear coefficient of the LCLV. The dynamics of the liquid crystals is much slower than the settling of the cavity field, thus, $E_{c}$ adiabatically follows the evolution of $n_{0}$ and $n_{1}$. By taking the wave propagation equation with the cavity boundary conditions, $E_{c}$ is expressed as a function of the scattering of $E_{p}$ onto the photo-induced refractive index grating through a complex operator accounting for the geometry of the cavity (inversion symmetry due to the odd number of mirrors), photon losses and phase retardation in a round trip.

Starting with a random initial condition, a transient speckle-like behaviour is observed, then a symmetry breaking occurs leading to an inhomogeneous build-up of the field showing large deviations from Gaussianity. The numerical PDF of the cavity field intensity are displayed in Figure 11 for different values of the pump. The tails are increasingly populated as the pump intensity increases, in agreement with the experimental observations. The numerical PDF are also well fitted by the stretched exponential function Equation (16).

\subsection{Mean-field hypercycle amplification model}

The inversion symmetry around the y axis induces an inhomogeneous build-up of the field, hence, spatial domains are coupled with other and distant domains. Because of this mechanism, large loops of amplification are introduced between distant domains, leading to a 
(a)

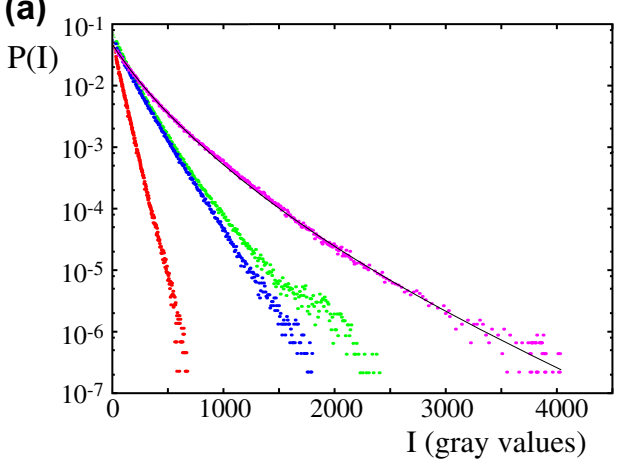

(b)

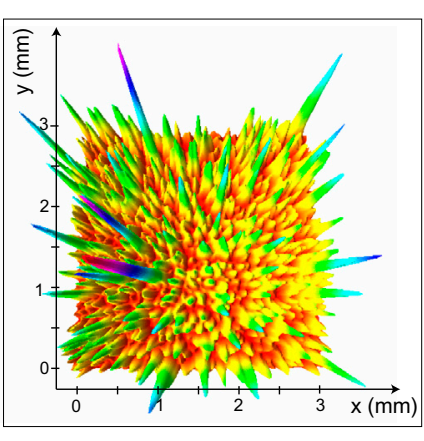

Figure 10. (a) PDF of the cavity field intensity; the non-linearity is increased from the steepest to the shallowest distribution; the black line is a fit with the stretched exponential function. (b) An instantaneous profile of the cavity field intensity. Reproduced with permission from [61].

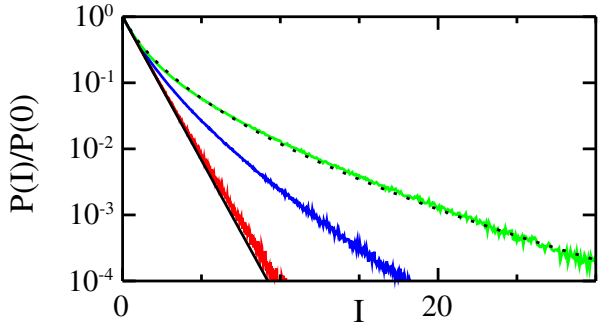

Figure 11. Numerical PDF of the cavity field intensity for different values of the pump $I_{p} / I_{\text {th }}=6$ (black), 8 (red), 10 (green); $1-\Gamma=$ 0.7. The dotted line is a fit with a stretched exponential, $c_{1}=3.73$. Reproduced with permission from [61].

focusing effect for which at some space locations the cavity field grows much more with respect to the surrounding places. To elucidate this mechanism, a simple two-mode model has been proposed, where only the evolution of the average refractive index $n_{1}$ is kept, the average being performed over the transverse $(x, y)$ plane. For a nearly plane cavity, $\bar{n}_{1}$ satisfies an equation of the form

$$
\tau \frac{\partial \bar{n}_{1}}{\partial t}=-\bar{n}_{1}+I_{p} F\left(\bar{n}_{1}\right)
$$

where the first and second terms account for the liquid crystal relaxation and, respectively, the grating feeding provided by the pump and cavity fields. A cubic function is taken as an ansatz for $F$, which describes a linear growth followed by saturation, this last one being mainly due to multiple scattering processes [62].

The above mean-field picture is modified by the nonlocal coupling due to the inversion of the $y$-axis after a round trip. Therefore, two mean fields $\bar{n}_{+}$and $\bar{n}_{-}$ must be considered, where the averages are performed over the upper and lower half-planes, respectively, and where each grating $\bar{n}_{ \pm}$is fed, after a round trip, by the grating $\bar{n}_{\mp}$ at the opposite side. The main features of the hypercycle amplification can be captured by using the cubic function $F\left(\bar{n}_{ \pm}\right)=\bar{n}_{ \pm}-\bar{n}_{ \pm}^{3}$. In this case, it is easy to show that for $1<I_{p}<2$ the system has only one stable solution, $\bar{n}_{-}=\bar{n}_{+}=0$, whereas for $I_{p} \geq 2$ a bifurcation occurs with the birth of the two stable asymmetric states breaking the $y \rightarrow-y$ symmetry.

Finally, if the non-locality is removed by changing the geometrical configuration of the resonator, the statistics becomes Gaussian again, demonstrating the importance of the nonlocal coupling in the generation of optical rogue waves $[5,61]$. The above simplified meanfield model qualitatively account for the experimental and numerical observations obtained for the non-linear cavity. It must be noted that hypercycle amplification is a mechanism of a general nature and that it plays a relevant role in different contexts, for instance, in autocatalytic systems or in spontaneous self-organisation. In the context of ocean rogue waves hypercycle amplification could play a role, for example, in the interaction between winds and currents.

\subsection{Multimode optical fiber experiments}

In order to investigate the relative role of granularity and inhomogeneity in the generation of optical rogue waves, a multimode optical fibre experiments has been set-up [63]. The input beam, from a frequency doubled solid-state laser, wavelength $532 \mathrm{~nm}$, is focused by a large numerical aperture lens on the entrance of a multimode fibre with $0.4 \mathrm{~mm}$ core diameter and $2 \mathrm{~m}$ length. Less than $1 \mathrm{~mW} / \mathrm{cm}^{2}$ is coupled inside the fibre. The intensity profile of the input beam is controlled by a computer driven spatial-light-modulator, SLM, by setting onto the SLM appropriate transmittance masks $T(x, y)$. While an uniform mask $T(x, y)$ allows the whole cone of input wave vectors to be coupled into the fibre, an inhomogeneous mask with a black hole prevents the wave vectors passing in that direction to be coupled into the fibre, thus providing a symmetry breaking mechanism which induces at the output domains of different average intensity. 

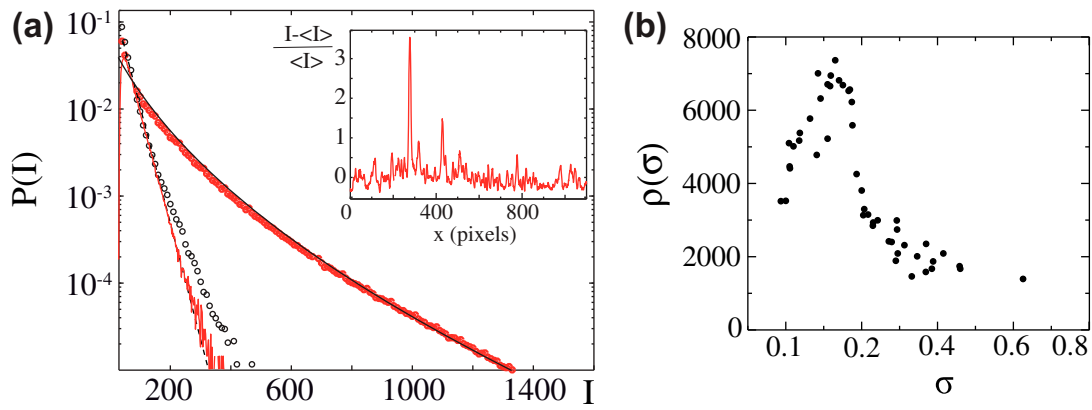

Figure 12. (a) PDF of the intensity at the output of the multimode fiber; solid (red) line: homogeneous mask; (black) empty dots: inhomogeneous mask; (red) filled dots: inhomogeneous mask and temporal perturbations; the black dashed line is a fit with an exponential function; the black solid line is a fit with a stretched exponential $\left(c_{1}=10, c_{2}=0.092\right)$. In the inset: $1 \mathrm{D}$ intensity profile $I(x)$ taken from a corresponding image; $\langle I\rangle$ is the intensity averaged over the whole image. (b) measured distribution of the variance $\sigma$ of the single PDF measured over each homogeneous domain at the output of the optical fiber.

Besides, the average size of the individual intensity grains of the fibre output is given by the total number of modes supported by the fibre, which is proportional to $(a / \lambda)^{2}$, with $a$ the radius of the fibre core and $\lambda$ the wavelength of light in air [54]. For the typical experimental conditions, the total number of modes can be estimated as $\sim 10^{6}$. Therefore, the intensity distribution at the fiber output reproduces a situation with a high number of individual grains clustered into large-scale spatial domains of different average intensity.

The PDFs of the intensity are determined by acquiring with a CCD camera a large set of images and then performing the histograms of the intensity on the whole image stack. The results are shown in Figure 12(a), where the PDF recorded for a uniform $T(x, y)$ is compared with the PDF recorded in the presence of an inhomogeneous mask. The first one (red solid line) is well fitted by an exponential function (black dashed line), as expected for speckles [54]. The second one (empty black dots) shows appreciable, though not too large, deviations from the exponential. In the same figure, a PDF with strong deviations from the exponential is also plotted; it is obtained by applying to the fibre a lateral perturbation $n(t)$ with a Gaussian distribution of the amplitude. The perturbation is realised by placing a piezoelectric emitter in contact with a side of the fibre. Thanks to the elasto-optical effect, when the piezo emits a low frequency acoustic wave, it locally modifies the optical paths inside the fibre, giving rise to a different spatial distribution of the output intensity, therefore, the detector collects events over different speckle configurations.

The symmetry breaking mechanism, together with the temporal perturbations, lead to the appearance of high amplitude peaks on the output intensity distributions $I(x, y)$. Correspondingly, the PDF of the intensity is largetailed and well described by the stretched exponential distribution, Equation (16). In the inset of Figure 12(a) a one-dimensional intensity profile taken along a $1 \mathrm{D}$ line of a corresponding image is displayed for the inhomogeneous case. A large amplitude peak, optical rogue wave, can be clearly distinguished.

In comparison with the non-linear optical cavity, here, the minimal size of the optical grains is due to the diffraction limit of the input beam and nonlocal coupling is provided by the asymmetric mask. On the other hand, in the cavity there is a spontaneous symmetry breaking and the average size of the individual optical grains is a result of the multimode dynamics. Then, in the nonlinear cavity the multimode dynamics spontaneously induces a continuous change of the cavity field, while here it is the time-dependent mechanical perturbation that introduces a dynamical behaviour forcing the change of speckle pattern configurations.

In both the linear and non-linear system the inhomogeneity plays a crucial role in determining the stretched exponential character of the PDF. In order to account for its effect, one can consider that at the fibre exit the intensity is distributed as a collection of speckle patterns made of domains with different average intensities. Within a single domain the statistics is exponential but the variance changes from domain to domain. As a consequence, the overall PDF becomes a stretched exponential as the events are counted over the whole field. More precisely, the stretched distribution can be obtained as a statistical mixture of many exponential distributions with different variance,

$$
P(I)=\int_{0}^{\infty} \mathrm{d} \sigma \rho(\sigma) \frac{e^{-I / \sigma}}{\sigma}
$$

where $\sigma$ is the local variance of the PDF in a single domain. The stretched distribution is exactly obtained when the distribution of $\sigma$ takes the form

$$
\rho(\sigma)=\sqrt{\sigma} e^{-\frac{c_{2} \sigma}{4}-\frac{c_{1}}{c_{2} \sigma}}
$$

Qualitatively, this prediction is well confirmed by the experimental results, where $\rho(\sigma)$ has been evaluated by 

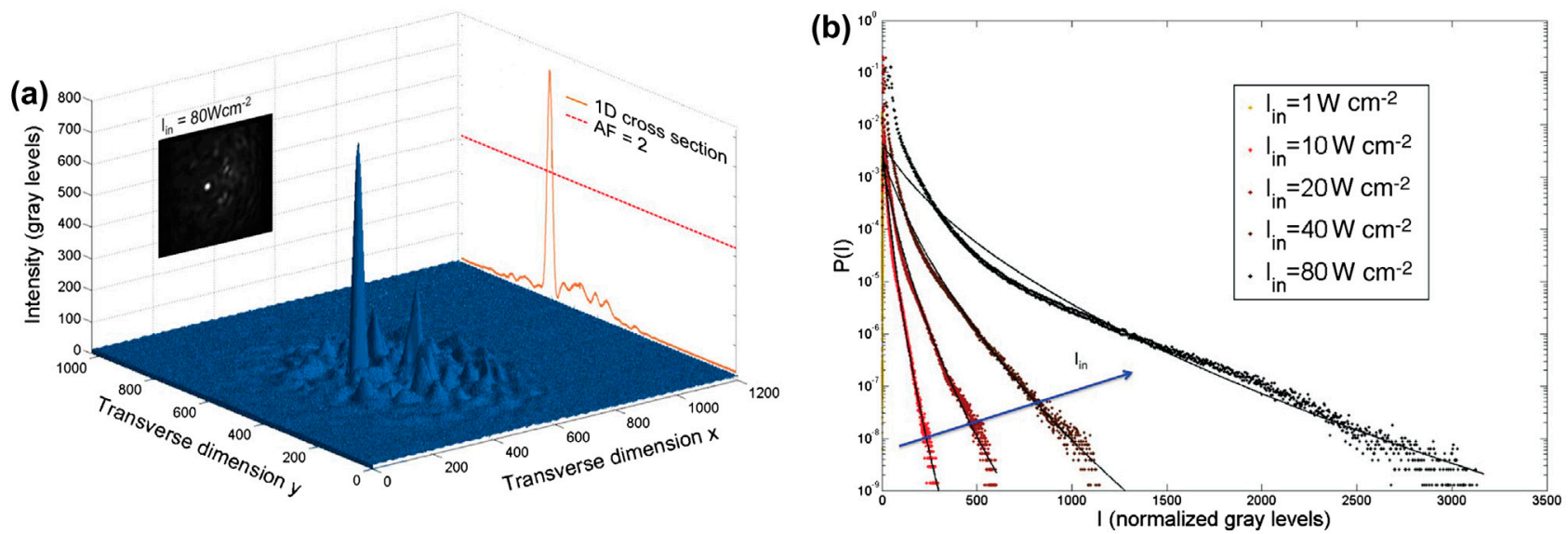

Figure 13. (a) Near-field distribution intensity of the backward beam: 2D intensity distribution in the transverse plane, 3D reconstruction and 1D profile. (b) PDF of the intensity in the presence of rogue waves; the curves are fitted with a stretched exponential; the deviations from the exponential behaviour increase with increasing the input intensity $I_{\text {in }}$. (From Ref. [65]).

performing the PDF analysis over small spatial domains and, then, by determining the local variance of the associated fit [63]. In Figure 12(b) the distribution $\rho(\sigma)$ of the measured local variance $\sigma$ is plotted for various values of $\sigma$, these being evaluated on the single PDF when measured over each homogeneous domain at the output of the fiber. A qualitative agreement with the theoretical expression, Equation (20), can be appreciated.

\section{Optical rogue waves in pattern-forming systems}

Optical rogue waves also occur in pattern-forming systems such as photorefractive or Kerr media with optical feedback under certain ranges of parameter. Rogue waves appear as erratic and high intensity peaks on the transverse optical wavefront, live for a lifetime dictated by the response time of the non-linear medium and exhibit rogue wave statistics of the intensity [64-67].

In a photorefractive crystal with a single-mirror feedback optical rogue waves appear by increasing the input light intensity after the first bifurcation to an ordered pattern state [65]. For increasing non-linearity a large number of unstable modes are brought above threshold, giving rise to a regime of spatiotemporal turbulence. In this regime, rare and intense peaks that exhibit the signatures of optical rogue waves have been identified in the transverse profile of the backward beam. The number of rogue waves increases with the increase of the pump intensity and, correspondingly, the PDF of the intensity evolves from exponential to a large-tailed distribution. As an example, in Figure 13 are shown a near field distribution of the backward beam intensity and a plot of the PDF of the intensity for increasing the input intensity $I_{\text {in }}$. It can be noted that the PDFs are well fitted by the stretched exponential, Equation (16).
Optical rogue waves have also been reported for Kerrlike media, as in a 1D system formed by a tilted nematic liquid crystal layer with a single-mirror feedback [66] and, more recently, in a liquid-crystal light valve subjected to optical feedback [67]. In both systems the rogue waves appear in the highly non-linear regime where many modes coexist. The rogue wave regime is identified by the appearance of intense localised peaks in transverse patterns as well as by the development of a supercontinuum in the spatial frequency domain [66] and the appearance of spatiotemporal chaos [67].

Finally, optical rogue waves have also been observed when a photorefractive ferroelectric crystal undergoes a structural phase transition [68]. Resolved micrometric bright spots with intensity more than 20 times that of the average have been reported. The transmitted spatial light distribution contains bright localised spots of anomalously large intensity that follow a large-tailed statistics. In this context, numerical simulations of a $2 \mathrm{D}+1$ generalised NLSE, containing a Kerr-saturated and a saturated Raman-like component, show that dynamics of soliton fusions and scale invariance play an important role in the observed rogue wave intensities and statistics.

\subsection{Optical filamentation and rogue wave phenomena}

Optical filamentation occurs when a light beam propagates in a non-linear medium characterised by a self-focusing non-linearity, hence, under certain circumstances, the transverse spread due to diffraction is counteracted by the self-focusing effect and the beam propagates with its transverse profile unaltered until losses take over the effect. The emergence of high amplitude optical filaments has been reported in a one-dimensional liquid crystal experiment, in which an optical wave 
turbulence [69] regime has been realised [70]. A laser beam is shaped as a laminar beam and propagates longitudinally inside a liquid crystal layer. Using a spatial light modulator, an initial condition is imposed in the form of waves at a spatial scale intermediate between the cell thickness and the dissipation length of the liquid crystal and with spatially random phases. The focusing non-linearity is provided by the orientational Kerr-like effect in the bulk of the liquid crystal layer. After the inverse cascade, leading to a smoothing of the initial beam profile, a modulation instability sets in, which is followed by the formation of filaments out of the random wave field. The subsequent evolution shows the merging of filaments and the development of a few and large amplitude filaments [70,71]. Correspondingly, the PDF of the intensity taken along the cell propagation distance show an evolution from the exponential behaviour at short propagation distance to large deviations from Gaussianity at large propagation distance [71].

Optical filaments are also observed in a xenon gas cell experiment, where their transient merging appears as a mechanism at the origin of optical rogue waves [72]. The filaments originate from the balance of linear diffraction, dispersion and non-linear Kerr effect in the gas cell. The rogue waves are isolated in space and in time. Their macroscopic origin is attributed to the local refractive index variations inside the non-linear medium, while microscopically, mergers between filaments appear to play a decisive role in the observed rogue wave statistics.

\section{Conclusions}

Since their observation on the ocean surfaces, rogue waves have become a paradigm for multidisciplinary physics and have been largely studied. Rogue waves have been reproduced as genuine space-time phenomena in a number of different experimental contexts. Hydrodynamics and optical experiments have particularly contributed to the current understanding of these complex phenomena, together with the continuous developments of mathematical models and numerical simulations in the field. A hierarchy of higher order solutions of the NLSE has been identified, where the prototype rogue wave solutions can be identified. Several of these solutions have been faithfully reproduced in laboratory experiments.

At the same time experimental and numerical studies have identified the non-Gaussian behaviour of the wave amplitude as one of the distinctive features of the rogue wave phenomena. Another characteristic of rogue waves is their erratic behaviour and their short lifetime. The emergence of individual grains of activity and, then, the grain clustering in inhomogeneous spatial domains have been reported in different types of optical experiments. In particular, in spatially extended optical systems the PDF of the intensity has been shown to evolve to a stretched exponential in the rogue wave regime. A non-Gaussian statistics of the amplitude accompanied by the formation of large amplitude filaments have also been observed in optical filamentation.

All these results suggest that the presence of a large number of coupled waves is at the basis of the rogue wave phenomenon. Non-local coupling also appear as an essential ingredient. However, a common definition of rogue wave criteria or a common classification of different classes of rogue waves are still a matter of study and constitute an active field of current fundamental investigations.

As for the real-world problems, there are several outcomes that stem from the current understanding of the rogue wave phenomena and that can be used for practical applications. For instance, the BFI index, Equation (2), is a parameter that can be employed for the rogue wave forecasting. A better understanding of the statistics of the wave amplitude can lead to a safer design of platforms or ships. There are, however, several open problems, for instance the role of the wind should be elucidated in order to provide in the future an even higher impact on practical applications. On a more basic level, the physical mechanism of formation of rogue waves in the ocean is still very much debated in the community, see for example [73] where it is conjectured that the modulational instability does not play a role in the formation of rogue waves in the real ocean.

\section{Acknowledgements}

We acknowledge useful discussions with P. Janssen and with N. Akhmediev. M.O. was supported by MIUR grant no. PRIN 2012BFNWZ2. M.O. thanks Dr B. Giulinico for fruitful discussions.

\section{Disclosure statement}

No potential conflict of interest was reported by the authors.

\section{Funding}

This work was supported by MIUR [grant number PRIN 2012BFNWZ2].

\section{Notes on contributors}

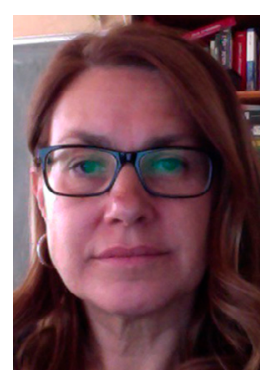

S. Residori is currently appointed as a Research Director of the Centre National de la Recherche Scientifique (CNRS) at the Institut Non Linéaire de Nice (INLN) in Nice, France. She received a Laurea Degree in Physics at the University of Bologna, Italy, and a $\mathrm{PhD}$ in Non-linear Optics at the University of Florence. She is head of the Optics of Liquid Crystals Group at INLN. Her research interests 
include soft matter, liquid crystals, optical wave mixing, optical vortices and light propagation in disordered media.

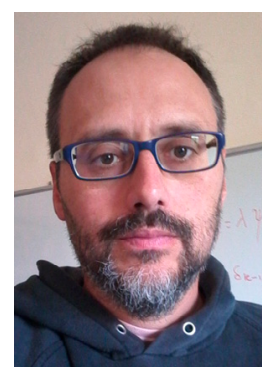

M. Onorato is Associate Professor at the Physics Department of the University of Torino, Italy. He graduated in Physics at the University of Torino and obtained a PhD in Fluid Mechanics from the Politecnico of Torino. His main research interests are ocean waves, wave turbulence, solitons and Bose Einstein condensates. He has been appointed as visiting professor in different universities such as Swinburne University, Australia, University of Science and Technology of Lille 1, France; Katholieke Universiteit Leuven, Belgium and Université Pierre et Marie Curie, France.

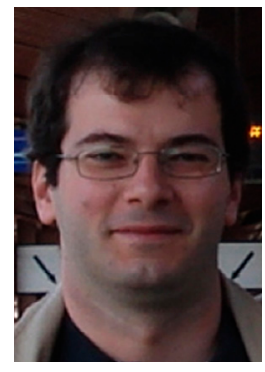

U. Bortolozzo is Associate Professor at the University of Nice-Sophia Antipolis, France. He has got a $\mathrm{PhD}$ in Complex Systems at the University of Florence (Italy) and in Physics at University of Nice-Sophia Antipolis (France). After his thesis he has been a postdoctoral researcher at the Ecole Normale Supérieure in Paris. He joined the University of Nice-Sophia Antipolis in 2009. His main research interests include optics, liquid crystals, soft matter, dynamical holography, non-linear dynamics and adaptive optics. He is senior member of the Optical Society.

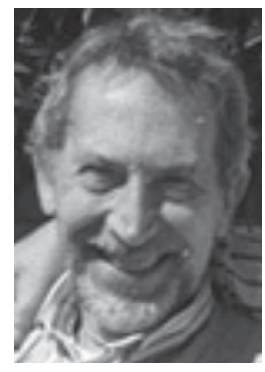

F. T. Arecchi is Professor Emeritus of Physics, University of Firenze and associate scientist, Istituto Nazionale di Ottica. Arecchi has been active in experimental and theoretical laser physics since the 1960s, first at CISEMilano-Italy, then from 1970 as full university professor (up to 1976 at University of Pavia, after that at the University of Firenze, where he was also appointed as head of Istituto Nazionale di Ottica). As a researcher, in 1965 Arecchi introduced the photon statistics as a tool to characterise coherence and laser fluctuations; in 1982 he provided the first evidence of deterministic chaos in lasers and then studied different dynamical behaviours introducing (1984) the classification of lasers into class A, B and C and (from 1990 on) exploring different control and synchronisation methods. In the 1990s he investigated the pattern formation in extended optical media, providing the first evidences of optical vortices and optical quasi-crystals. Since 2000, Arecchi is also investigating the physics of cognitive processes. He is author and co-author of more than 400 scientific papers, plus 12 books.

\section{References}

[1] S. Haver, Evidences of the existence of freak waves, in Rogue Waves 2000: Proceedings of a workshop organized by Ifremer and held in Brest, France, 29-30 November 2000, within de Brest SeaTechWeek 2000, Vol. 32, Editions Quae, 2001, p. 129.
[2] E.M. Bitner-Gregersen, L. Fernández, J.M. Lefèvre, J. Monbaliu, and A. Toffoli, The north sea Andrea storm and numerical simulations, Nat. Hazards Earth Syst. Sci. 14(6) (2014), pp. 1407-1415.

[3] N. Mori, P.C. Liu, and T. Yasuda, Analysis of freak wave measurements in the sea of Japan, Ocean Eng. 29(11) (2002), pp. 1399-1414.

[4] D.R. Solli, C. Ropers, P. Koonath, and B. Jalali, Optical rogue waves, Nature 450(7172) (2007), pp. 1054-1057.

[5] M. Onorato, S. Residori, U. Bortolozzo, A. Montina, and F.T. Arecchi, Rogue waves and their generating mechanisms in different physical contexts, Phys. Rep. 528(2) (2013), pp. 47-89.

[6] J.M. Dudley, F. Dias, M. Erkintalo, and G. Genty, Instabilities, breathers and rogue waves in optics, Nat. Photonics 8(10) (2014), pp. 755-764.

[7] P.A.E.M. Janssen, On some consequences of the canonical transformation in the Hamiltonian theory of water waves, J. Fluid Mech. 637(1) (2009), pp. 1-44.

[8] M. Onorato, A.R. Osborne, and M. Serio, On Deviations from Gaussian Statistics for Surface Gravity Waves, in Rogue Waves, Proceedings Hawaiian Winter Workshop, University of Hawaii at Manoa, USA. 2005. arXiv preprint nlin/0503071.

[9] A.R. Osborne, Nonlinear Ocean Waves, Academic Press, 2010.

[10] N.N. Akhmediev, V.M. Eleonskii, and N.E. Kulagin, Exact first-order solutions of the nonlinear Schrödinger equation, Theor. Math. Phys. 72(2) (1987), pp. 809-818.

[11] A.R. Osborne, M. Onorato, and M. Serio, The nonlinear dynamics of rogue waves and holes in deep-water gravity wave train, Phys. Lett. A 275 (2000), pp. 386-393.

[12] D.H. Peregrine, Water waves, nonlinear Schrödinger equations and their solutions, J. Aust. Math. Soc. Ser. B 25(1) (1983), pp. 16-43.

[13] M. Tajiri and Y. Watanabe, Breather solutions to the focusing nonlinear Schrödinger equation, Phys. Rev. E 57 (1998), pp. 3510-3519.

[14] V.E. Zakharov and A.A. Gelash, Nonlinear stage of modulation instability, Phys. Rev. Lett. 111 (2013), p. 054101.

[15] M. Onorato, A.R. Osborne, M. Serio, and S. Bertone, Freak wave in random oceanic sea states, Phys. Rev. Lett. 86(25) (2001), pp. 5831-5834.

[16] P.A.E.M. Janssen, Nonlinear four-wave interaction and freak waves, J. Phys. Ocean. 33(4) (2003), pp. 863-884.

[17] A.C. Newell and J.V. Moloney, Nonlinear Optics, Addison-Wesley, Redwood City, CA, 1992.

[18] A. Chabchoub, B. Kibler, C. Finot, G. Millot, M. Onorato, J.M. Dudley, and A.V. Babanin, The nonlinear Schrödinger equation and the propagation of weakly nonlinear waves in optical fibers and on the water surface, Ann. Phys. 361 (2015), pp. 490-500.

[19] E.A. Kuznetsov, Solitons in a parametrically unstable plasma, Akademiia Nauk SSSR Doklady 236 (1977), pp. 575-577.

[20] Y.C. Ma, The perturbed plane-wave solutions of the cubic Schrödinger equation, Stud. Appl. Math. 60 (1979), pp. 43-58.

[21] N. Akhmediev, A. Ankiewicz, and M. Taki, Waves that appear from nowhere and disappear without a trace, Phys. Lett. A 373(6) (2009), pp. 675-678. 
[22] V.I. Shrira and V.V. Geogjaev, What makes the Peregrine soliton so special as a prototype of freak waves? J. Eng. Math. 67(1) (2010), pp. 11-22.

[23] K.B. Dysthe and K. Trulsen, Note on breather type solutions of the NLS as models for freak-waves, Phys. Scripta T82 (1999), pp. 48-52.

[24] A.A. Gelash and V.E. Zakharov, Superregular solitonic solutions: A novel scenario for the nonlinear stage of modulation instability, Nonlinearity 27(4) (2014), p. R1.

[25] B. Kibler, A. Chabchoub, A. Gelash, N. Akhmediev, and V.E. Zakharov, Superregular breathers in optics and hydrodynamics: Omnipresent modulation instability beyond simple periodicity, Phys. Rev. X 5(4) (2015), p. 041026.

[26] N.N. Akhmediev, V.M. Eleonskii, and N.E. Kulagin, Generation of periodic trains of picosecond pulses in an optical fiber: Exact solutions, Sov. Phys. JETP 62(5) (1985), pp. 894-899.

[27] N. Akhmediev, A. Ankiewicz, and J.M. Soto-Crespo, Rogue waves and rational solutions of the nonlinear Schrödinger equation, Phys. Rev. E 80(2) (2009), p. 026601.

[28] N.N. Akhmediev and A. Ankiewicz, Solitons: Nonlinear Pulses and Beams, Chapman \& Hall, London, 1997.

[29] D.J. Kedziora, A. Ankiewicz, and N. Akhmediev, Classifying the hierarchy of nonlinear-Schrödingerequation rogue-wave solutions, Phys. Rev. E 88 (2013), p. 013207.

[30] A. Chabchoub, N.P. Hoffmann, and N. Akhmediev, Rogue wave observation in a water wave tank, Phys. Rev. Lett. 106(20) (2011), p. 204502.

[31] M. Onorato, D. Proment, G. Clauss, and M. Klein, Rogue waves: From nonlinear Schrödinger breather solutions to sea-keeping test, PloS one 8(2) (2013), p. e54629.

[32] A. Chabchoub, N. Hoffmann, M. Onorato, and N. Akhmediev, Super rogue waves: observation of a higherorder breather in water waves, Phys. Rev. X 2 (2012), p. 011015.

[33] A. Chabchoub, B. Kibler, J.M. Dudley, and N. Akhmediev, Hydrodynamics of periodic breathers, Philos. Trans. Roy. Soc. London A: Math. Phys, Eng. Sci. 372(2027) (2014), p. 20140005.

[34] A. Chabchoub, N.P. Hoffmann, M. Onorato, A. Slunyaev, A. Sergeeva, E. Pelinovsky, and N. Akhmediev, Observation of a hierarchy of up to fifth-order rogue waves in a water tank, Phys. Rev. E 86 (2012), p. 056601.

[35] B. Kibler, J. Fatome, C. Finot, G. Millot, F. Dias, G. Genty, et al., The Peregrine soliton in nonlinear fibre optics, Nat. Phys. 6(10) (2010), pp. 790-795.

[36] A. Bendahmane, A. Mussot, P. Szriftgiser, O. Zerkak, G. Genty, J.M. Dudley, and A. Kudlinski, Experimental dynamics of Akhmediev breathers in a dispersion varying optical fiber, Opt. Lett. 39(15) (2014), pp. 4490-4493.

[37] B. Kibler, J. Fatome, C. Finot, G. Millot, G. Genty, B. Wetzel, et al., Observation of Kuznetsov-Ma soliton dynamics in optical fibre, Sci. Rep. 2 (2012), Article No: 463.

[38] S. Chen, J.M. Soto-Crespo, and P. Grelu, Watch-handlike optical rogue waves in three-wave interactions, Opt. Express 23(1) (2015), pp. 349-359.

[39] L. Cavaleri, L. Bertotti, L. Torrisi, E. Bitner-Gregersen, M. Serio, M. Onorato, Rogue waves in crossing seas: The Louis
Majesty accident, J. Geophys. Res.: Oceans (1978-2012). 117(C11) (2012), p. 8.

[40] M. Onorato, Extreme waves in random crossing seas: Laboratory experiments and numerical simulations, Geophys. Res. Lett. 38(6) (2011), p. 5.

[41] M. Onorato, D. Proment, and A. Toffoli, Freak waves in crossing seas, Eur. Phys. J. Spec. Topics 185(1) (2010), pp. 45-55.

[42] M. Onorato, A.R. Osborne, and M. Serio, Modulational instability in crossing sea states: A possible mechanism for the formation of freak waves, Phys. Rev. Lett. 96(1) (2006), p. 014503.

[43] P.K. Shukla, I. Kourakis, B. Eliasson, M. Marklund, and L. Stenflo, Instability and evolution of nonlinearly interacting water waves, Phys. Rev. Lett. 97(9) (2006), p. 094501.

[44] V.P. Ruban, Giant waves in weakly crossing sea states, J. Exp. Theor. Phys. 110(3) (2010), pp. 529-536.

[45] F. Baronio, A. Degasperis, M. Conforti, and S. Wabnitz, Solutions of the vector nonlinear Schrödinger equations: evidence for deterministic rogue waves, Phys. Rev. Lett. 109(4) (2012), p. 044102.

[46] F. Baronio, M. Conforti, A. Degasperis, S. Lombardo, M. Onorato, and S. Wabnitz, Vector rogue waves and baseband modulation instability in the defocusing regime, Phys. Rev. Lett. 113(3) (2014), p. 034101.

[47] F. Baronio, S. Chen, P. Grelu, S. Wabnitz, and M. Conforti, Baseband modulation instability as the origin of rogue waves, Phys. Rev. A 91(3) (2015), p. 033804.

[48] F. Baronio, M. Conforti, A. Degasperis, and S. Lombardo, Rogue waves emerging from the resonant interaction of three waves, Phys. Rev. Lett. 111(11) (2013), p. 114101.

[49] S. Chen, J.M. Soto-Crespo, and P. Grelu, Dark threesister rogue waves in normally dispersive optical fibers with random birefringence, Opt. Express 22(22) (2014), pp. 27 632-27 642 .

[50] K. Wing Chow, H. Ning Chan, D.J. Kedziora, and R.H.J. Grimshaw, Rogue wave modes for the long wave-short wave resonance model, J. Phys. Soc. Japan 82(7) (2013), p. 074001.

[51] B. Kinsman, Wind Waves, Prentice-Hall, New York, 1965.

[52] P.A.E.M. Janssen, The Interaction of Ocean Waves and Wind, Cambridge University Press, Cambridge, 2004.

[53] W.H. Munk, Proposed uniform procedure for observing waves and interpreting instrument records, in SIO Wave Project, Translations by D.D. Bidde and R.L. Wiegel can be found in translations of four French papers on ocean wave climate distribution functions. 1944.

[54] J.W.Goodman, Speckle Phenomena in Optics: Theory and Applications, Roberts and Company Publishers, Greenwood Village, CO, USA, 2007, .

[55] P. Walczak, S. Randoux, and P. Suret, Optical rogue waves in integrable turbulence, Phys. Rev. Lett. 114(14) (2015), p. 143903.

[56] P. Suret, R.E. Koussaifi, A. Tikan, C. Evain, S. Randoux, C. Szwaj, and S. Bielawski, Direct observation of rogue waves in optical turbulence using time microscopy, (2016). Available at arXiv preprint arXiv:1603.01477.

[57] M. Conforti, A. Mussot, J. Fatome, A. Picozzi, S. Pitois, C. Finot, M. Haelterman, B. Kibler, C. Michel, and G. Millot, Turbulent dynamics of an incoherently pumped passive optical fiber cavity: Quasisolitons, dispersive waves, and extreme events, Phys. Rev. A 91(2) (2015), p. 023823. 
[58] M. Onorato, D. Proment, G. El, S. Randoux, and P. Suret, On the origin of heavy-tail statistics in equations of the nonlinear Schrödinger type, Phys. Lett. A 380(39) (2016), pp. 3173-3177.

[59] S.Y. Annenkov and V.I. Shrira, Modelling transient sea states with the generalised kinetic equation, in Rogue and Shock Waves in Nonlinear Dispersive Media, M. Onorato, S. Residori, and F. Baronio, eds., Springer International Publishing, 2016.

[60] U. Bortolozzo, A. Montina, F.T. Arecchi, J.P. Huignard, and S. Residori, Spatiotemporal pulses in a liquid crystal optical oscillator, Phys. Rev. Lett. 99(2) (2007), pp. 3-6.

[61] A. Montina, U. Bortolozzo, S. Residori, and F.T. Arecchi, Non-Gaussian statistics and extreme waves in a nonlinear optical cavity, Phys. Rev. Lett. 103(17) (2009), p. 173901.

[62] A. Montina, U. Bortolozzo, S. Residori, J.P. Huignard, and F. Arecchi, Complex dynamics of a unidirectional optical oscillator based on a liquid-crystal gain medium, Phys. Rev. A 76(3) (2007), pp. 1-14.

[63] F.T. Arecchi, U. Bortolozzo, A. Montina, and S. Residori, Granularity and inhomogeneity are the joint generators of optical rogue waves, Phys. Rev. Lett. 106(15) (2011), p. 153901.

[64] P.M. Lushnikov and N. Vladimirova, Non-Gaussian statistics of multiple filamentation, Opt. Lett. 35(12) (2010), pp. 1965-1967.

[65] N. Marsal, V. Caullet, D. Wolfersberger, and M. Sciamanna, Spatial rogue waves in a photorefractive pattern-forming system, Opt. Lett. 39(12) (2014), pp. 3690-3693.
[66] E. Louvergneaux, V. Odent, M.I. Kolobov, and M. Taki, Statistical analysis of spatial frequency supercontinuum in pattern forming feedback systems, Phys. Rev. A 87 (2013), p. 063802 .

[67] M.G. Clerc, G. González-Cortés, and M. Wilson, Extreme events induced by spatiotemporal chaos in experimental optical patterns, Opt. Lett. 41(12) (2016), pp. 2711-2714.

[68] D. Pierangeli, F. Di Mei, C. Conti, A.J. Agranat, and E. DelRe, Spatial rogue waves in photorefractive ferroelectrics, Phys. Rev. Lett. 115(9) (2015), p. 093901.

[69] S. Dyachenko, A.C. Newell, A. Pushkarev, and V.E. Zakharov, Optical turbulence: Weak turbulence, condensates and collapsing filaments in the nonlinear Schrödinger equation, Phys. D 57(1) (1992), pp. 96-160.

[70] U. Bortolozzo, J. Laurie, S. Nazarenko, and S. Residori, Optical wave turbulence and the condensation of light, J. Opt. Soc. Amer. B 26(12) (2009), pp. 2280-2284.

[71] J. Laurie, U. Bortolozzo, S. Nazarenko, and S. Residori, One-dimensional optical wave turbulence: experiment and theory, Phys. Rep. 514 (2012), pp. 121-175.

[72] S. Birkholz, E.T.J. Nibbering, C. Brée, S. Skupin, A. Demircan, G. Genty, and G. Steinmeyer, Spatiotemporal rogue events in optical multiple filamentation, Phys. Rev. Lett. 111 (2013), p. 243903.

[73] F. Fedele, J. Brennan, S.P. De León, J. Dudley, and F. Dias, Real world ocean rogue waves explained without the modulational instability, Sci. Rep. 6 (2016), p. 11. 